\title{
Dynamical conductivity of lithium-intercalated hexagonal boron nitride films: A memory function approach
}

\author{
Zoran Rukelj]* \\ Department of Physics, University of Fribourg, 1700 Fribourg, Switzerland \\ and Department of Physics, Faculty of Science, University of Zagreb, Bijenička c. 32, 10000 Zagreb, Croatia
}

(Received 25 June 2020; revised 14 September 2020; accepted 12 October 2020; published 9 November 2020)

\begin{abstract}
This work is a study of dynamical conductivity of a quasi-two-dimensional heterostructure $\mathrm{Li}(\mathrm{BN})_{8}$. The conducting electrons have a free-electron-like parabolic dispersion and are assumed to scatter only on acoustic phonons. The approach used to derive the generalized Drude relation with frequency and temperature dependent relaxation consists of defining the induced current density as a function of the electron-hole operator. This element is proportional to the perturbating electric field and is decomposed into the zeroth and second order of the electron-phonon interaction. The electron-hole operator is then evaluated using the Heisenberg equation. The connection between the electron-hole operator and the memory function is shown. The analytical properties of the imaginary and real part of the frequency and temperature dependent memory function, that describe electron scattering on acoustic and optical phonons, are examined in detail. Finally, the dynamical conductivity of $\mathrm{Li}(\mathrm{BN})_{8}$ is calculated, with the explanation of all relevant features originating from the memory function.
\end{abstract}

DOI: 10.1103/PhysRevB.102.205108

\section{INTRODUCTION}

Recently, a new class of structurally stable quasi-2D conductors were presented [1-4] with a free-electron-like conduction band. These systems are realized as three layered crystal heterostructures held together by the van der Waals interaction. They are comprised of an atomically thin lithium (Li) metallic layer sandwiched between two wide band gap [5] boron nitride (BN) insulating layers. The stoichiometric formula for this particular compound is $\operatorname{Li}(\mathrm{BN})_{8}$. This material is used here as a test system for the development of a general Drude conductivity formula. It has been chosen due to the simple shape of the half-field parabolic conduction band [4].

In this study, the scattering on acoustic and optical phonons is taken to be the only relaxation mechanism of conducting electrons. The goal is to find a general form of the intraband conductivity formula, also called generalized Drude formula [6]. The main part of the generalized Drude formula is the complex relaxation function which is frequency $(\omega)$ and temperature $(T)$ dependent. Usually, this relaxation function is called the memory function. It was originally introduced by Zwanzig and Mori [7-9] and first calculated by Götze and Wölfle $[6,10,11]$ by evaluating the current-current correlation function [12] using the equation of motion technique. Here, it is derived using a similar equation of motion approach for the electron-hole operator [13-15], also known as the density matrix [16-19]. The electron-hole operator which is proportional to the perturbating field is divided in two contributions; the zeroth and the second order of electronphonon interaction. Both the zeroth and the second order

*zrukelj@phy.hr contributions are evaluated using the Heisenberg equation. It is the second order electron-phonon contribution to the total electron-hole operator that is proportional to the momentum and frequency dependent function. This function is identified to be a memory function. Finally induced current, defined by this electron-hole operator, gives the intraband conductivity tensor. Other approaches [20-25] derive a similar function. An excellent overview of these methods [26] compares their features and limitations. The memory function derived here implements the two main contributions to the electron-hole operator dynamics. The first is the phonon mediated electronhole scattering, also known as the current vertex correction, which results in a reduction of the forward scattering and is usually given as the $1-\cos \varphi$ term in the collision integral in the standard transport equations [27]. The second contribution is the single particle energy renormalization. It consists of the electron and hole self-energy change due to interaction with phonons. The resulting generalized Drude formula reproduces the well known Bloch temperature dependent dc resistivity [6,28,29], while for $\omega \neq 0$ it qualitatively follows the results obtained by Götze and Wölfle [10].

The advantage of the equation of motion technique for the electron-hole operator is the ease of inclusion of additional scattering mechanisms, like electron impurity [30], impurity mediated electron phonon [31,32], electron magnon [33,34], or electron electron [35-37]. A chainlike evaluation of the commutators in the Heisenberg equation makes every step of the evaluation easily traceable. The inclusion of higher order contributions, for example, the fourth order in electron-phonon interaction, follows from the continuation of the chained evaluation of the operators within the Heisenberg equation. Naturally, in the Heisenberg picture, the operators and the response functions, like conductivity, are evaluated in the frequency domain. The results presented in this article 


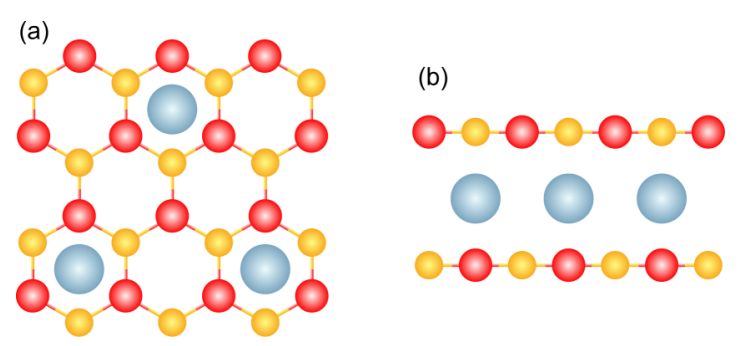

FIG. 1. (a) Top view and (b) side view of the three layered structure $\mathrm{Li}(\mathrm{BN})_{8}$. The boron, nitrogen, and lithium atoms are represented by the colors red, yellow, and blue, respectively. The distance between the Li atoms is $a=5 \AA$, while the thickness of the structure is $6.4 \AA$. The two BN layers are shifted with respect to each other, so that the boron atom in the bottom $\mathrm{BN}$ layer lies below a nitrogen atom in the top BN layer and vice versa.

are a finite $\omega$ version of the static Fermi-rule approach to the electron-phonon scattering problem in two-dimensional conductors [38-40].

This paper is structured as follows. In Sec. II, the system properties are specified, consisting of the electron and phonon Hamiltonian, as well as the electron coupling to the scalar potential and to phonons. In Sec. III, the electron-hole operator is defined and evaluated using the Heisenberg equation. The memory function is derived together with the generalized Drude formula. Section IV is reserved for the analytical evaluation of the imaginary part of the memory function, which is then further evaluated for the specific cases of acoustic and optical phonons. Additionally, the real part of the memory function is derived using the Kramers-Kronig relations. Finally, the $(\omega, T)$-dependent conductivity of $\mathrm{Li}(\mathrm{BN})_{8}$ is calculated and analyzed.

\section{HAMILTONIAN OF Li(BN)}

The structure of the $\mathrm{Li}(\mathrm{BN})_{8}$ is depicted in Fig. 1 as seen from the top and from the side. The conducting electrons in $\mathrm{Li}(\mathrm{BN})_{8}$ are described by a single band Hamiltonian [4]

$$
\hat{H}_{e l}=\sum_{\mathbf{k} \sigma} \varepsilon_{\mathbf{k}} c_{\mathbf{k} \sigma}^{\dagger} c_{\mathbf{k} \sigma} .
$$

The dispersion is $\varepsilon_{\mathbf{k}}=\hbar^{2} k^{2} / 2 m^{*}$ with the effective mass $m^{*}=1.15 m_{e}$. Other parameters are Fermi wave vector $k_{F}=$ $0.537 \AA^{-1}$, the corresponding Fermi energy $\varepsilon_{F}=0.96 \mathrm{eV}$ as measured from the bottom of the conduction band, and the primitive cell volume $v_{p c}=a^{2} \sqrt{3} / 2$, where $a=5 \AA$.

In the $\mathbf{A}^{\text {tot }}=0$ gauge, the charge-field interaction Hamiltonian is given by the coupling of total scalar potential $\Phi^{\text {tot }}(\kappa, \omega)$ to the electron charge density

$$
\hat{H}_{\text {field }}=\frac{1}{V} \sum_{\kappa} \sum_{\mathbf{k} \sigma} e \Phi^{\mathrm{tot}}(\boldsymbol{\kappa}, \omega) c_{\mathbf{k} \sigma}^{\dagger} c_{\mathbf{k}+\kappa \sigma} .
$$

Further down, the total longitudinal electric field is used instead. The Cartesian field components $\alpha$ are given by the screened scalar potential $E_{\alpha}(\kappa, \omega)=-i \kappa_{\alpha} \Phi^{\text {tot }}(\kappa, \omega)$. The phonon Hamiltonian with the phonon frequencies $\omega_{\mathbf{q}}^{\lambda}$ is

$$
\hat{H}_{\mathrm{ph}}=\sum_{\lambda, \mathbf{q}} \hbar \omega_{\mathbf{q}}^{\lambda} b_{\lambda \mathbf{q}}^{\dagger} b_{\lambda \mathbf{q}},
$$

where, for simplicity, $\lambda$ stands for polarization and the phonon type. The electron-phonon interaction Hamiltonian is

$$
\hat{H}_{\mathrm{el}-\mathrm{ph}}=\frac{1}{\sqrt{N}} \sum_{\mathbf{k}, \mathbf{q}, \sigma} D_{\mathbf{q}}^{\lambda} c_{\mathbf{k}+\mathbf{q} \sigma}^{\dagger} c_{\mathbf{k} \sigma}\left(b_{\lambda \mathbf{q}}+b_{-\lambda \mathbf{q}}^{\dagger}\right) .
$$

In the above expression, $N$ is the number of the primitive cells in the crystal. A simple long wavelength approximation is used for $D_{\mathbf{q}}^{\lambda}$ for acoustical $(A)$ and optical $(O)$ phonons. This approximation gives

$$
\left|D_{\mathbf{q}}^{\lambda}\right|^{2} \approx D_{\lambda} \hbar \omega_{\mathbf{q}}^{\lambda}
$$

where $\lambda \in(A, O)$. Constants $D_{\lambda}$ are defined later in the text. The choice of coupling elements Eq. (2.5) is backed by numerous $a b$ initio studies of electron-phonon coupling in two dimensional systems [41,42]. The general property is that the square of the coupling element for acoustic phonons is roughly linear with the phonon momenta, while for the nonpolar optical phonon it is a constant.

\section{GENERALIZED DRUDE CONDUCTIVITY}

\section{A. Electron-hole operator}

The basic element of the semiclassical Landau transport equations is the nonequilibrium momentum distribution function $n_{\mathbf{k}, \kappa}(\omega)$ [43]. This function $n_{\mathbf{k}, \kappa}(\omega)$ depends on the electron or hole momentum $\mathbf{k}$, as well as the wave vector and the frequency $(\kappa, \omega)$ of the external perturbation. In the linear response theory it is decomposed as

$$
n_{\mathbf{k}, \kappa}(\omega)=n_{\mathbf{k}}+\delta n_{\mathbf{k}, \kappa}(\omega) .
$$

$n_{\mathbf{k}}$ is the equilibrium distribution function or momentum distribution function which is approximated by the Fermi-Dirac distribution $f_{\mathbf{k}}$ [44]. $\delta n_{\mathbf{k}, \kappa}(\omega)$ is proportional to the perturbation field and will be addressed as a electron-hole operator. In a quantum field theory an analog to $\delta n_{\mathbf{k}, \kappa}(\omega)$ would be a two-particle Green function or a particle-hole propagator, with a Bethe-Salpeter equation governing its dynamics [45].

Denoting the ground state average and the frequency dependence as \langle\rangle$_{0}$ and \langle\rangle$_{\omega}$, respectively, both $n_{\mathbf{k}}$ and $\delta n_{\mathbf{k}, \kappa}(\omega)$ can be written using fermion operators: $n_{\mathbf{k}}=\left\langle c_{\mathbf{k} \sigma}^{\dagger} c_{\mathbf{k} \sigma}\right\rangle_{0}$ and $\delta n_{\mathbf{k}, \kappa}(\omega)=\left\langle c_{\mathbf{k} \sigma}^{\dagger} c_{\mathbf{k}+\kappa \sigma}\right\rangle_{\omega}$. Brackets \langle\rangle$_{\omega}$ also denote the nonequilibrium average, which is zero in absence of the external perturbation. The induced current density is defined as

$$
\begin{aligned}
J_{\alpha}(\boldsymbol{\kappa}, \omega) & =\frac{1}{V} \sum_{\mathbf{k} \sigma} e v_{\alpha \mathbf{k}} \delta n_{\mathbf{k}, \boldsymbol{\kappa}}(\omega) \\
& =\sigma_{\alpha \alpha}(\boldsymbol{\kappa}, \omega) E_{\alpha}(\boldsymbol{\kappa}, \omega)
\end{aligned}
$$

where $v_{\alpha \mathbf{k}}=(1 / \hbar) \partial \varepsilon_{\mathbf{k}} / \partial k_{\alpha}$ is the $\alpha$ component of electron group velocity. $\delta n_{\mathbf{k}, \kappa}(\omega)$ is determined by Heisenberg equation

$$
i \hbar \frac{\partial}{\partial t} \delta n_{\mathbf{k}, \kappa}(\omega)=\left\langle\left[c_{\mathbf{k} \sigma}^{\dagger} c_{\mathbf{k}+\kappa \sigma}, \hat{H}\right]\right\rangle_{\omega} .
$$

$\hat{H}$ is the total Hamiltonian of the system, containing all the contributions mentioned in Sec. II. In accordance with the perturbative approach to the electron-phonon scattering, $\delta n_{\mathbf{k}, \kappa}(\omega)$ 

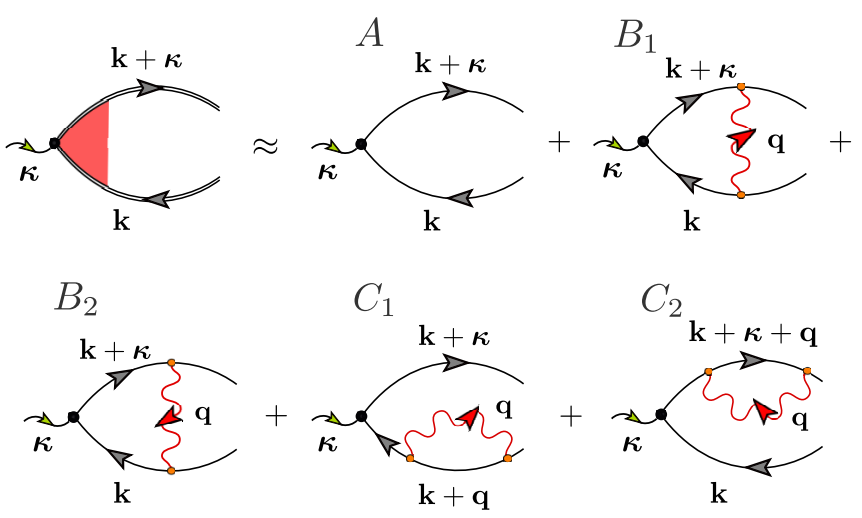

FIG. 2. Diagrammatic representation of an expansion of the fully dressed irreducible electron-hole operator. Diagram $A$ is the zero order and diagrams $B_{1}$ to $C_{2}$ are the second order of the electronphonon interaction denoted by a red wavy line. $C_{1}$ and $C_{2}$ describe the electron and hole single-particle self-energies. $B_{1}$ and $B_{2}$ describe the current vertex renormalization or phonon mediated electron-hole scattering.

is decomposed as follows:

$$
\delta n_{\mathbf{k}, \kappa}(\omega) \approx \delta n_{\mathbf{k}, \kappa}^{(0)}(\omega)+\delta n_{\mathbf{k}, \kappa}^{(2)}(\omega) .
$$

The superscript indices in Eq. (3.4) stand for the order of electron-phonon interaction (2.4). Also, $\delta n_{\mathbf{k}, \kappa}(\omega)$ can be represented diagrammatically, as shown in Fig. 2, and calculated by Matsubara method [46,47]. In the next two subsections, the electron-hole operator (3.4) is evaluated under the assumption that the phonon subsystem remains in equilibrium. Otherwise, a coupled set of electron and phonon nonequilibrium distributions like (3.1) has to be solved [14].

\section{B. Zeroth order contribution $\delta n^{(0)}$}

To make the derivation as simple as possible, quantum numbers like $\sigma$ and $\lambda$ (spin and polarization) have been omitted during the procedure, and are inserted into the final result. The Hamiltonian in Eq. (3.3) consists of $\hat{H}=\hat{H}_{e l}+\hat{H}_{\text {field }}$. The standard change to the time derivative is $i \hbar \partial / \partial t \rightarrow \hbar \omega+$ $i \eta$, where $\eta$ is a small adiabatic parameter. The commutators are evaluated using the rules given in Appendix A. The commutator containing electron Hamiltonian (2.1) is

$$
\left\langle\left[c_{\mathbf{k}}^{\dagger} c_{\mathbf{k}+\kappa}, \hat{H}_{e l}\right]\right\rangle_{\omega}=\left(\varepsilon_{\mathbf{k}+\kappa}-\varepsilon_{\mathbf{k}}\right) \delta n_{\mathbf{k}, \kappa}(\omega)
$$

Similarly, the field term has been evaluated using the random phase approximation [27]

$$
\left\langle\left[c_{\mathbf{k}}^{\dagger} c_{\mathbf{k}+\kappa}, \hat{H}_{\text {field }}\right]\right\rangle_{\omega} \approx\left(f_{\mathbf{k}}-f_{\mathbf{k}+\kappa}\right) \frac{i e}{\kappa_{\alpha}} E_{\alpha}(\kappa, \omega) .
$$

Thus Eq. (3.3) with the commutation results (3.5) and (3.6) gives $\delta n_{\mathbf{k}, \kappa}(\omega)$, which is of zero order in $\hat{H}_{\mathrm{el}-\mathrm{ph}}$

$$
\begin{aligned}
\delta n_{\mathbf{k}, \kappa}^{(0)}(\omega) & =\frac{f_{\mathbf{k}}-f_{\mathbf{k}+\kappa}}{\hbar \omega+\varepsilon_{\mathbf{k}}-\varepsilon_{\mathbf{k}+\kappa}+i \eta} \frac{i e}{\kappa_{\alpha}} E_{\alpha}(\boldsymbol{\kappa}, \omega) \\
& \approx-\frac{i e \hbar v_{\alpha \mathbf{k}}}{\hbar \omega+i \eta} \frac{\partial f_{\mathbf{k}}}{\partial \varepsilon_{\mathbf{k}}} E_{\alpha}(\omega) .
\end{aligned}
$$

In the second row of the previous expression, the numerator has been expanded to the first order in $\kappa_{\alpha}$ and the energy difference in the denominator is neglected. If the conductivity Eq. (3.2) is calculated using the zeroth order part of the electron-hole operator in Eq. (3.7), a simple Drude formula is obtained

$$
\sigma_{\alpha \alpha}(\omega) \approx \frac{i e^{2}}{m_{e}} \frac{n_{\alpha}}{\omega+i \gamma}
$$

with

$$
n_{\alpha}=-\frac{1}{V} \sum_{\mathbf{k} \sigma} m_{e} v_{\alpha \mathbf{k}}^{2} \frac{\partial f_{\mathbf{k}}}{\partial \varepsilon_{\mathbf{k}}}=\frac{m_{e} \varepsilon_{F}}{\pi \hbar^{2}}
$$

denoting the effective concentration of the intraband charge carriers. For a parabolic band with the effective mass components $m_{\alpha}^{*}$, a simple connection between the effective and total concentration of electrons $n$ and the bare electron mass $m_{e}$ can be written as $n_{\alpha} / m_{e}=n / m_{\alpha}^{*}$. Also, the $\eta$ term in the denominator is usually generalized to a finite constant relaxation $\eta \rightarrow \hbar \gamma=\hbar / \tau$.

\section{Second order contribution $\delta n^{(2)}$}

To get to the second order of the electron-phonon contribution to the electron-hole operator, the phonon Hamiltonian Eq. (2.3) and electron-phonon Hamiltonian Eq. (2.4) are added in Eq. (3.3). So, in addition to the already evaluated commutators Eqs. (3.5) and (3.6), there are four terms with mixed fermion and boson operators

$$
\left\langle\left[c_{\mathbf{k}}^{\dagger} c_{\mathbf{k}+\kappa}, \hat{H}_{\mathrm{el}-\mathrm{ph}}\right]\right\rangle_{\omega}=\frac{1}{\sqrt{N}} \sum_{\mathbf{q}} D_{\mathbf{q}}^{*}\left(\left\langle c_{\mathbf{k}}^{\dagger} c_{\mathbf{k}+\kappa-\mathbf{q}} b_{\mathbf{q}}\right\rangle_{\omega}+\left\langle c_{\mathbf{k}}^{\dagger} c_{\mathbf{k}+\kappa-\mathbf{q}} b_{-\mathbf{q}}^{\dagger}\right\rangle_{\omega}-\left\langle c_{\mathbf{k}+\mathbf{q}}^{\dagger} c_{\mathbf{k}+\kappa} b_{\mathbf{q}}\right\rangle_{\omega}-\left\langle c_{\mathbf{k}+\mathbf{q}}^{\dagger} c_{\mathbf{k}+\kappa} b_{-\mathbf{q}}^{\dagger}\right\rangle_{\omega}\right) .
$$

The operators in the above expression are further evaluated using the Heisenberg equation which does not contain $\hat{H}_{\text {field }}$ due to being in the linear response regime where no higher order field terms are necessary. For illustration, the Heisenberg equation is explicitly solved for the first operator in Eq. (3.10) with the analogous procedure for the rest of them. The bare energy part is trivial

$$
\left\langle\left[c_{\mathbf{k}}^{\dagger} c_{\mathbf{k}+\kappa-\mathbf{q}} b_{\mathbf{q}}, \hat{H}_{e l}+\hat{H}_{\mathrm{ph}}\right]\right\rangle_{\omega}=\left(\varepsilon_{\mathbf{k}+\kappa-\mathbf{q}}-\varepsilon_{\mathbf{k}}+\hbar \omega_{\mathbf{q}}\right)\left\langle c_{\mathbf{k}}^{\dagger} c_{\mathbf{k}+\kappa-\mathbf{q}} b_{\mathbf{q}}\right\rangle_{\omega},
$$

while the commutator with the electron-phonon interaction becomes increasingly complex

$$
\begin{aligned}
\left\langle\left[c_{\mathbf{k}}^{\dagger} c_{\mathbf{k}+\kappa-\mathbf{q}} b_{\mathbf{q}}, \hat{H}_{\mathrm{el}-\mathrm{ph}}\right]\right\rangle_{\omega}= & \frac{1}{\sqrt{N}} \sum_{\mathbf{k}^{\prime}} D_{\mathbf{q}}\left\langle c_{\mathbf{k}}^{\dagger} c_{\mathbf{k}+\kappa-\mathbf{q}} c_{\mathbf{k}^{\prime}-\mathbf{q}}^{\dagger} c_{\mathbf{k}^{\prime}}\right\rangle_{\omega}+\frac{1}{\sqrt{N}} \sum_{\mathbf{q}^{\prime}} D_{\mathbf{q}^{\prime}}^{*}\left\langle c_{\mathbf{k}}^{\dagger} c_{\mathbf{k}+\kappa-\mathbf{q}-\mathbf{q}^{\prime}}\left(b_{\mathbf{q}^{\prime}}+b_{-\mathbf{q}^{\prime}}^{\dagger}\right) b_{\mathbf{q}}\right\rangle_{\omega} \\
& -\frac{1}{\sqrt{N}} \sum_{\mathbf{q}^{\prime}} D_{\mathbf{q}^{\prime}}^{*}\left\langle c_{\mathbf{k}+\mathbf{q}^{\prime}}^{\dagger} c_{\mathbf{k}+\kappa-\mathbf{q}}\left(b_{\mathbf{q}^{\prime}}+b_{-\mathbf{q}^{\prime}}^{\dagger}\right) b_{\mathbf{q}}\right\rangle_{\omega} .
\end{aligned}
$$


Here, the mean field approximation of averaging in the ground state \langle\rangle$_{0}$ is applied. For the first term on the right hand side of Eq. (3.12), containing four fermion operators, mean field averaging gives

$$
\begin{aligned}
& \left\langle c_{\mathbf{k}}^{\dagger} c_{\mathbf{k}+\kappa-\mathbf{q}} c_{\mathbf{k}^{\prime}-\mathbf{q}}^{\dagger} c_{\mathbf{k}^{\prime}}\right\rangle_{0} \\
& \approx c_{\mathbf{k}}^{\dagger} c_{\mathbf{k}^{\prime}} \delta_{\mathbf{k}^{\prime}, \mathbf{k}+\kappa}\left(1-f_{\mathbf{k}+\kappa-\mathbf{q}}\right)-c_{\mathbf{k}-\mathbf{q}}^{\dagger} c_{\mathbf{k}+\kappa-\mathbf{q}} \delta_{\mathbf{k}^{\prime}, \mathbf{k}} f_{\mathbf{k}} .
\end{aligned}
$$

Similarly, the boson operators are also averaged

$$
\left\langle\left(b_{\mathbf{q}^{\prime}}+b_{-\mathbf{q}^{\prime}}^{\dagger}\right) b_{\mathbf{q}}\right\rangle_{0}=N\left(\omega_{\mathbf{q}}\right) \delta_{\mathbf{q},-\mathbf{q}^{\prime}},
$$

where $N\left(\omega_{\mathbf{q}}\right)$ is the Bose-Einsten distribution. Hence the commutator (3.12) reduces to

$$
\begin{aligned}
& \left\langle\left[c_{\mathbf{k}}^{\dagger} c_{\mathbf{k}+\kappa-\mathbf{q}} b_{\mathbf{q}}, \hat{H}_{\mathrm{el}-\mathrm{ph}}\right]\right\rangle_{\omega} \\
& \approx \frac{1}{\sqrt{N}} D_{\mathbf{q}}\left[1-f_{\mathbf{k}+\kappa-\mathbf{q}}+N\left(\omega_{\mathbf{q}}\right)\right] \delta n_{\mathbf{k}, \kappa}(\omega) \\
& \quad-\frac{1}{\sqrt{N}} D_{\mathbf{q}}\left[f_{\mathbf{k}}+N\left(\omega_{\mathbf{q}}\right)\right] \delta n_{\mathbf{k}-\mathbf{q}, \kappa}(\omega) .
\end{aligned}
$$

The above result, together with the commutator (3.11), provides the solution to the first operator in Eq. (3.10),

$$
\begin{aligned}
& \left\langle c_{\mathbf{k}}^{\dagger} c_{\mathbf{k}+\kappa-\mathbf{q}} b_{\mathbf{q}}\right\rangle_{\omega} \\
& \approx-\frac{1}{\sqrt{N}} D_{\mathbf{q}} \frac{f_{\mathbf{k}}+N\left(\omega_{\mathbf{q}}\right)}{\hbar \omega+\varepsilon_{\mathbf{k}}-\varepsilon_{\mathbf{k}+\kappa-\mathbf{q}}-\hbar \omega_{\mathbf{q}}+i \eta} \delta n_{\mathbf{k}-\mathbf{q}, \kappa}(\omega) \\
& \quad+\frac{1}{\sqrt{N}} D_{\mathbf{q}} \frac{1-f_{\mathbf{k}+\kappa-\mathbf{q}}+N\left(\omega_{\mathbf{q}}\right)}{\hbar \omega+\varepsilon_{\mathbf{k}}-\varepsilon_{\mathbf{k}+\kappa-\mathbf{q}}-\hbar \omega_{\mathbf{q}}+i \eta} \delta n_{\mathbf{k}, \kappa}(\omega) .
\end{aligned}
$$

The same procedure is applied for the remaining three operators in Eq. (3.10). Finally, all evaluated commutators are assembled back into the Heisenberg equation (3.3), yielding an equation for the electron-hole operator $\delta n_{\mathbf{k}, \kappa}(\omega)$ :

$$
\begin{aligned}
(\hbar \omega & \left.+\varepsilon_{\mathbf{k}}-\varepsilon_{\mathbf{k}+\kappa}+i \eta\right) \delta n_{\mathbf{k}, \kappa}(\omega) \\
= & \left(f_{\mathbf{k}}-f_{\mathbf{k}+\kappa}\right) \frac{i e}{\kappa_{\alpha}} E_{\alpha}(\boldsymbol{\kappa}, \omega)+\sum_{\mathbf{q}} \delta n_{\mathbf{k}, \kappa}(\omega) \\
& \times \frac{1}{N} \sum_{s= \pm 1}\left|D_{\mathbf{q}}\right|^{2}\left(\frac{s\left[f_{\mathbf{k}+\kappa+\mathbf{q}}+N\left(s \omega_{\mathbf{q}}\right)\right]}{\hbar \omega+\varepsilon_{\mathbf{k}}-\varepsilon_{\mathbf{k}+\kappa+\mathbf{q}}+s \hbar \omega_{\mathbf{q}}+i \eta}\right. \\
& \left.+\frac{s\left[f_{\mathbf{k}+\mathbf{q}}+N\left(s \omega_{\mathbf{q}}\right)\right]}{\hbar \omega+\varepsilon_{\mathbf{k}+\mathbf{q}}-\varepsilon_{\mathbf{k}+\kappa}-s \hbar \omega_{\mathbf{q}}+i \eta}\right) \\
- & \sum_{\mathbf{q}} \delta n_{\mathbf{k}+\mathbf{q}, \kappa}(\omega) \\
& \times \frac{1}{N} \sum_{s= \pm 1}\left|D_{\mathbf{q}}\right|^{2}\left(\frac{s\left[f_{\mathbf{k}}+N\left(s \omega_{\mathbf{q}}\right)\right]}{\hbar \omega+\varepsilon_{\mathbf{k}}-\varepsilon_{\mathbf{k}+\kappa+\mathbf{q}}-s \hbar \omega_{\mathbf{q}}+i \eta}\right. \\
& \left.+\frac{s\left[f_{\mathbf{k}+\kappa}+N\left(s \omega_{\mathbf{q}}\right)\right]}{\hbar \omega+\varepsilon_{\mathbf{k}+\mathbf{q}}-\varepsilon_{\mathbf{k}+\kappa}+s \hbar \omega_{\mathbf{q}}+i \eta}\right) .
\end{aligned}
$$

In order to write the expression (3.17) more compactly, a $\mathbf{q} \rightarrow-\mathbf{q}$ was changed wherever possible to maintain the same sign of $\mathbf{q}$ throughout Eq. (3.17). Additionally, the property of the Bose-Einstein distribution $-N\left(-\omega_{\mathbf{q}}\right)=1+N\left(\omega_{\mathbf{q}}\right)$ has been used. Now it is safe to put $\boldsymbol{\kappa} \approx 0$ in the energy dispersions in the above expression, except in the field part where $f_{\mathbf{k}+\kappa}$ is expanded to the first order, as in Eq. (3.7). With the aid of auxiliary sums $S_{1}$ and $S_{2}$, which contain the first and second $\sum_{s}$ contributions in Eq. (3.17), we have

$$
\begin{aligned}
(\hbar \omega & +i \eta) \delta n_{\mathbf{k}, \kappa}(\omega) \\
= & -i e \hbar v_{\alpha \mathbf{k}} \frac{\partial f_{\mathbf{k}}}{\partial \varepsilon_{\mathbf{k}}} E_{\alpha}(\omega)+\sum_{\mathbf{q}} \delta n_{\mathbf{k}, \kappa}(\omega) S_{1}(\mathbf{k}, \mathbf{q}, \omega) \\
& -\sum_{\mathbf{q}} \delta n_{\mathbf{k}+\mathbf{q}, \kappa}(\omega) S_{2}(\mathbf{k}, \mathbf{q}, \omega) .
\end{aligned}
$$

Dividing the above expression with $\hbar \omega+i \eta$, multiplying it by $v_{\alpha \mathbf{k}}$, and summing over $\mathbf{k}$ gives

$$
\begin{aligned}
& \sum_{\mathbf{k}} e v_{\alpha \mathbf{k}} \delta n_{\mathbf{k}, \kappa}(\omega)=\sum_{\mathbf{k}} e v_{\alpha \mathbf{k}} \delta n_{\mathbf{k}, \kappa}^{(0)}(\omega) \\
& +\sum_{\mathbf{k}} \sum_{\mathbf{q}} e v_{\alpha \mathbf{k}} \delta n_{\mathbf{k}, \kappa}(\omega) \frac{S_{1}(\mathbf{k}, \mathbf{q}, \omega)}{\hbar \omega+i \eta} \\
& -\sum_{\mathbf{k}} \sum_{\mathbf{q}} e v_{\alpha \mathbf{k}} \delta n_{\mathbf{k}+\mathbf{q}, \kappa}(\omega) \frac{S_{2}(\mathbf{k}, \mathbf{q}, \omega)}{\hbar \omega+i \eta} .
\end{aligned}
$$

In the third term on the right hand side of Eq. (3.19), the change in the sum $\mathbf{k} \rightarrow \mathbf{k}-\mathbf{q}$ followed by $\mathbf{q} \rightarrow-\mathbf{q}$ enables the factorization of Eq. (3.19)

$$
\begin{aligned}
\sum_{\mathbf{k}} e v_{\alpha \mathbf{k}} \delta n_{\mathbf{k}, \kappa}(\omega)= & \sum_{\mathbf{k}} e v_{\alpha \mathbf{k}} \delta n_{\mathbf{k}, \kappa}^{(0)}(\omega) \\
& -\sum_{\mathbf{k}} e v_{\alpha \mathbf{k}} \delta n_{\mathbf{k}, \kappa}(\omega) \frac{\hbar M_{\alpha}(\mathbf{k}, \omega)}{\hbar \omega+i \eta},
\end{aligned}
$$

where the $\mathbf{k}$ and $\alpha$ dependent memory function $M_{\alpha}(\mathbf{k}, \omega)$ is introduced:

$$
\begin{aligned}
\hbar M_{\alpha}(\mathbf{k}, \omega)= & \sum_{\mathbf{q}} S_{1}(\mathbf{k}, \mathbf{q}, \omega)-\frac{v_{\alpha \mathbf{k}+\mathbf{q}}}{v_{\alpha \mathbf{k}}} S_{2}(\mathbf{k}+\mathbf{q},-\mathbf{q}, \omega) \\
= & \frac{1}{N} \sum_{\mathbf{q}} \sum_{\lambda} \sum_{s, s^{\prime}= \pm 1}\left|D_{\mathbf{q}}^{\lambda}\right|^{2}\left(1-\frac{v_{\alpha \mathbf{k}+\mathbf{q}}}{v_{\alpha \mathbf{k}}}\right) \\
& \times \frac{s N\left(s \omega_{\mathbf{q}}^{\lambda}\right)+s f_{\mathbf{k}+\mathbf{q}}}{\hbar \omega+s^{\prime}\left(\varepsilon_{\mathbf{k}}-\varepsilon_{\mathbf{k}+\mathbf{q}}\right)+s s^{\prime} \hbar \omega_{\mathbf{q}}^{\lambda}+i \eta} .
\end{aligned}
$$

In the above definition of the memory function, the dependence on the phonon type and polarization has been restored. Equation (3.20) is the final result of the operator equation of motion analysis. However, it contains the electron-hole operator on both sides. This implies that the solution to Eq. (3.20) should be searched for self-consistently. The clue for obtaining an analytical solution is to compare Eqs. (3.4) and (3.20), considering the conditions on the electron hole propagator as outlined in Sec. III A. Since $M_{\alpha}(\mathbf{k}, \omega)$ is second order in electron-phonon interaction, we conclude that $\delta n_{\mathbf{k}, \kappa}(\omega) \approx$ $\delta n_{\mathbf{k}, \kappa}^{(0)}(\omega)$ on the right side of Eq. (3.20). Hence we obtain

$$
\delta n_{\mathbf{k}, \kappa}^{(2)}(\omega)=-\delta n_{\mathbf{k}, \kappa}^{(0)}(\omega) \frac{\hbar M_{\alpha}(\mathbf{k}, \omega)}{\hbar \omega+i \eta} .
$$


Naturally, a question arises regarding the interpretation of the memory function $M_{\alpha}(\mathbf{k}, \omega)$. To provide an answer, we inspect the electron self-energy as derived in Appendix B and compare it with Eq. (3.21). We define $\widetilde{\Sigma}_{\alpha}(\mathbf{k}, \omega)$ as an electron self-energy, Eq. (B5), in which the vertex part is included:

$$
\widetilde{\Sigma}_{\alpha}(\mathbf{k}, \omega)=\sum_{\mathbf{q}}\left(1-v_{\alpha \mathbf{k}+\mathbf{q}} / v_{\alpha \mathbf{k}}\right) \Sigma(\mathbf{q}, \mathbf{k}, \omega) .
$$

Then $M_{\alpha}(\mathbf{k}, \omega)$ as defined in Eq. (3.21) can be written as

$$
M_{\alpha}(\mathbf{k}, \omega)=\widetilde{\Sigma}_{\alpha}\left(\mathbf{k}, \omega+\varepsilon_{\mathbf{k}}\right)-\widetilde{\Sigma}_{\alpha}^{*}\left(\mathbf{k}, \varepsilon_{\mathbf{k}}-\omega\right) .
$$

The first self-energy in the above expression belongs to the electron and is defined with respect to the bare electron energy $\varepsilon_{\mathbf{k}}$. The second one belongs to the hole, since it is analytical in the lower complex plane $(-\omega-i \eta)$, and is also defined with respect to the bare energy $\varepsilon_{\mathbf{k}}$. Thus an important result is reached: the memory function is a sum of the electron and hole self-energies and the contribution to the self-energies originating from the electron-hole phonon mediated interaction [21].

Next, the dynamical conductivity is derived.

\section{Generalized Drude formula}

The zeroth and the second order in the electron-phonon expansion of the electron propagator Eqs. (3.7) and (3.22) are now known, and can be inserted into Eq. (3.2) to yield the conductivity tensor

$$
\begin{aligned}
\sigma_{\alpha \alpha}(\omega) & \approx \frac{i e^{2}}{m_{e}} \frac{1}{V} \sum_{\mathbf{k} \sigma}-\frac{\partial f_{\mathbf{k}}}{\partial \varepsilon_{\mathbf{k}}}\left(m_{e} v_{\alpha \mathbf{k}}^{2}\right)\left(\frac{1}{\omega+i \eta}-\frac{M_{\alpha}(\mathbf{k}, \omega)}{(\omega+i \eta)^{2}}\right) \\
& =\frac{i e^{2}}{m_{e}} \frac{n_{\alpha}}{\omega+i \eta}\left(1-\frac{M(\omega)}{\omega+i \eta}\right) .
\end{aligned}
$$

In the above expression, an averaged memory function over the Fermi surface is defined:

$$
M(\omega)=\frac{1}{n_{\alpha}} \frac{1}{V} \sum_{\mathbf{k} \sigma}-\frac{\partial f_{\mathbf{k}}}{\partial \varepsilon_{\mathbf{k}}}\left(m_{e} v_{\alpha \mathbf{k}}^{2}\right) M_{\alpha}(\mathbf{k}, \omega) .
$$

Recognizing Eq. (3.25) as the first and second term in high $\omega$ expansion of the conductivity, we can write

$$
\sigma_{\alpha \alpha}(\omega) \approx \frac{i e^{2}}{m_{e}} \frac{n_{\alpha}}{\omega+M(\omega)} .
$$

The above relation is called the generalized Drude formula. The same result has been obtained by Götze and Wölfle [10]. However, the memory function they obtained differs from Eq. (3.26), and the discrepancies can be assigned to a different derivation method. Causality requirements on the conductivity Eq. (3.27) impose constraints on the imaginary $M_{2}(\omega)=M_{2}(-\omega)$ and real $M_{1}(\omega)=-M_{1}(-\omega)$ part of the memory function Eq. (3.26). This further implies that the two parts are connected by the Kramers-Kronig relations.

\section{PROPERTIES OF THE MEMORY FUNCTION}

\section{A. Imaginary part of the memory function $M_{2}(\omega)$}

Here the imaginary part of the memory function (3.26) is evaluated. The evaluation is restricted to small temperature $k_{B} T \ll \varepsilon_{F}$ and frequency $\hbar \omega \sim \hbar \omega_{\mathbf{q}} \ll \varepsilon_{F}$ scales. Inserting
Eq. (3.21) into Eq. (3.26) and taking the imaginary part gives

$$
\begin{aligned}
M_{2}(\omega)= & \frac{2 \pi}{\hbar n_{\alpha} V N} \sum_{\mathbf{q}}\left|D_{\mathbf{q}}\right|^{2} \sum_{\mathbf{k}}-\frac{\partial f_{\mathbf{k}}}{\partial \varepsilon_{\mathbf{k}}} m_{e} v_{\alpha \mathbf{k}}^{2}\left(1-\frac{v_{\alpha \mathbf{k}+\mathbf{q}}}{v_{\alpha \mathbf{k}}}\right) \\
& \times \sum_{s, s^{\prime}= \pm 1} s\left(N\left(s \omega_{\mathbf{q}}\right)+f_{\mathbf{k}+\mathbf{q}}\right) \\
& \times \delta\left(\hbar \omega+s^{\prime}\left(\varepsilon_{\mathbf{k}}-\varepsilon_{\mathbf{k}+\mathbf{q}}\right)+s s^{\prime} \hbar \omega_{\mathbf{q}}\right) .
\end{aligned}
$$

Although written in the momentum representation, in Eq. (4.1) we recognize a transport version of the averaged electron-phonon spectral function $\alpha_{\mathrm{tr}}^{2} F(\omega)$ :

$$
\alpha_{\mathrm{tr}}^{2} F(\omega) \propto \sum_{\lambda \mathbf{q}}\left\langle\left.\left|D_{\mathbf{q}}^{\lambda}\right|^{2} \delta\left(\hbar \omega-\hbar \omega_{\mathbf{q}}^{\lambda}\right)\left(1-v_{\alpha \mathbf{k}+\mathbf{q}} / v_{\alpha \mathbf{k}}\right)\right|_{F S} .\right.
$$

This function is often used to describe the effect multiple phonon branches have on the scattering of conducting electrons [21,48-50]. The function $\alpha_{\mathrm{tr}}^{2} F(\omega)$ is averaged over the Fermi surface \langle\rangle$_{F S}$. As seen from Eq. (4.1), this Fermi surface averaging is naturally present in the form of the Fermi-Dirac distribution derivative, which in the case of small $T \ll \varepsilon_{F}$ restricts the summation over $\mathbf{k}$ to the Fermi surface. This condition fails in the low doped semiconductors [51], where a strong temperature dependence of the electron chemical potential completely smears the Fermi-Dirac distribution and its derivative. Since we know the phonon distributions, as well as the electron-phonon matrix elements, we proceed to evaluate Eq. (4.1) in the momentum representation. Only in the final expression do we switch to the energy representation.

Choosing $\alpha=x$, though the same result applies for $y$ direction as well, the velocity term in Eq. (4.1) becomes

$$
v_{\alpha \mathbf{k}}^{2}\left(1-v_{\alpha \mathbf{k}+\mathbf{q}} / v_{\alpha \mathbf{k}}\right)=-\left(\hbar / m^{*}\right)^{2} k_{x} q_{x} .
$$

Changing to the polar coordinates gives

$$
k_{x} q_{x}=k q\left[\cos \left(\varphi_{1}-\varphi_{2}\right)+\cos \left(\varphi_{1}+\varphi_{2}\right)\right] / 2,
$$

where $\varphi_{1}$ and $\varphi_{2}$ are polar angles for $\mathbf{k}$ and $\mathbf{q}$, respectively. Next, the energy conservation is implemented from the delta function in Eq. (4.1)

$$
\varepsilon_{\mathbf{k}+\mathbf{q}}=\varepsilon_{\mathbf{k}}+s^{\prime} \hbar \omega_{\mathbf{q}}+s \hbar \omega
$$

into the distribution functions, while at the same time removing the $\omega$ and $\omega_{\mathbf{q}}$ from the delta function argument when inspecting the momentum conservation, since $\hbar \omega \sim \hbar \omega_{\mathbf{q}} \ll$ $\varepsilon_{F}$. Inserting the $T \rightarrow 0$ property of the Fermi-Dirac function derivative and changing to integrals in Eq. (4.1), we get

$$
\begin{aligned}
M_{2}(\omega) \approx & \frac{v_{p c} \hbar m_{e}}{n_{\alpha} 16 \pi^{3} m^{* 2}} \int k^{2} d k \delta\left(\varepsilon_{\mathbf{k}}-\varepsilon_{F}\right) \int_{0}^{2 \pi} d \varphi_{1} \int_{0}^{q_{c}} q^{2} d q \\
& \times \int_{0}^{2 \pi} d \varphi_{2}\left|D_{\mathbf{q}}\right|^{2}\left[\cos \left(\varphi_{1}-\varphi_{2}\right)+\cos \left(\varphi_{1}+\varphi_{2}\right)\right] \\
& \times \delta\left(\varepsilon_{\mathbf{k}}-\varepsilon_{\mathbf{k}+\mathbf{q}}\right) \sum_{s, s^{\prime}= \pm 1} s\left[N\left(s \omega_{\mathbf{q}}\right)\right. \\
& \left.+f\left(\varepsilon_{\mathbf{k}}+s^{\prime} \hbar \omega+s \hbar \omega_{\mathbf{q}}\right)\right] .
\end{aligned}
$$

Here $q_{c}$ has been introduced as the upper limit of $q$ integration. The determination of $q_{c}$ has been left for the end of the section. The explicit forms of the $\delta$ functions in 

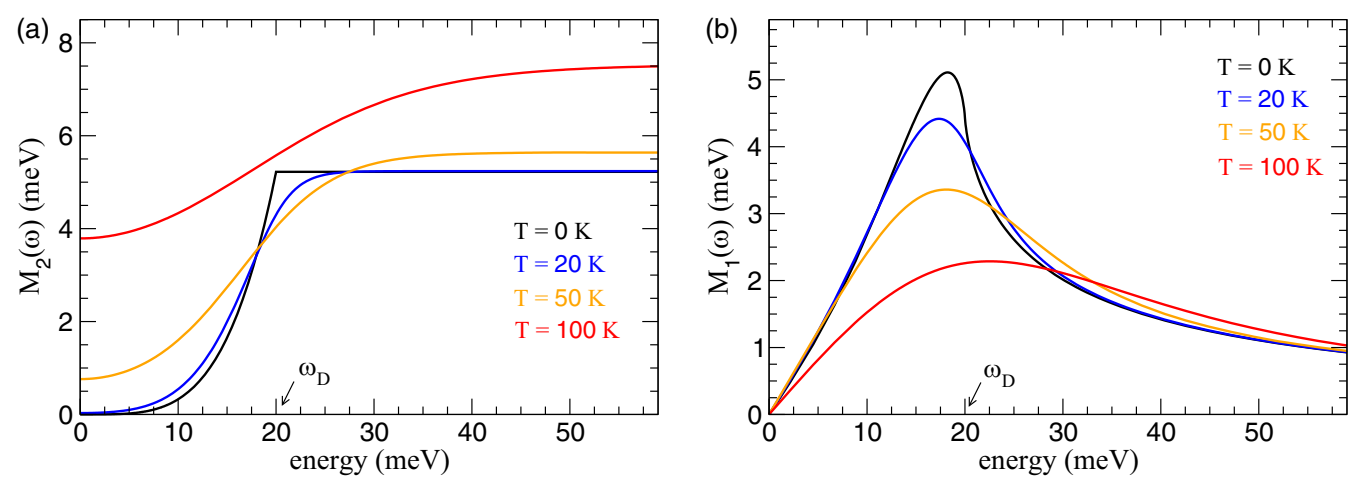

FIG. 3. (a) Imaginary and (b) real part of the memory function describing the scattering of an electron on an acoustic phonon. The real and imaginary parts of the memory function are calculated at various temperatures. The parameters used in the calculation are $\varepsilon_{F}=0.96 \mathrm{eV}$, sound speed $c=3500 \mathrm{~m} / \mathrm{s}, D_{A}=0.5 \mathrm{eV}$, and Debye energy $\hbar \omega_{D}=20 \mathrm{meV}$, with equivalent Debye temperature $T_{D}=230 \mathrm{~K}$. The imaginary part is calculated directly from Eq. (4.12), while the real part is obtained from Eq. (4.12) using the Kramers-Kronig relations.

expression (4.6) are

$$
\begin{gathered}
\delta\left(\varepsilon_{\mathbf{k}}-\varepsilon_{F}\right)=\frac{\delta\left(k-k_{F}\right)}{\hbar^{2} k_{F} / m^{*}}, \\
\delta\left(\varepsilon_{\mathbf{k}}-\varepsilon_{\mathbf{k}+\mathbf{q}}\right)=\delta\left(\frac{\hbar^{2} q^{2}}{2 m^{*}}+\frac{\hbar^{2}}{m^{*}} k q \cos \left(\varphi_{1}-\varphi_{2}\right)\right) .
\end{gathered}
$$

Changing the angular variables to $\varphi_{1}-\varphi_{2}=\vartheta$ and $\varphi_{1}+$ $\varphi_{2}=2 \varphi_{1}-\vartheta$, the expression (4.6) becomes

$$
\begin{aligned}
& M_{2}(\omega) \\
& \approx \\
& \quad \frac{v_{p c} k_{F} m_{e}}{n_{\alpha} \hbar 16 \pi^{3} m^{*}} \int_{0}^{2 \pi} d \varphi_{1} \int_{0}^{q_{c}} q^{2} d q\left|D_{\mathbf{q}}\right|^{2} \int_{0}^{2 \pi} d \vartheta(\cos \vartheta \\
& \left.\quad+\cos 2 \varphi_{1} \cos \vartheta+\sin 2 \varphi_{1} \sin \vartheta\right) \delta\left(\frac{\hbar^{2} q^{2}}{2 m^{*}}+\frac{\hbar^{2}}{m^{*}} k_{F} q \cos \vartheta\right) \\
& \quad \times \sum_{s, s^{\prime}= \pm 1} s\left[N\left(s \omega_{\mathbf{q}}\right)+f\left(\varepsilon_{F}+s^{\prime} \hbar \omega+s \hbar \omega_{\mathbf{q}}\right)\right] .
\end{aligned}
$$

The integration over $\varphi_{1}$ vanishes, leaving only

$$
\int_{0}^{2 \pi} d \vartheta \cos \vartheta \delta\left(\frac{\hbar^{2} q^{2}}{2 m^{*}}+\frac{\hbar^{2}}{m^{*}} k_{F} q \cos \vartheta\right)=\frac{1 /\left(2 \varepsilon_{F}\right)}{\sqrt{1-\left(q / 2 k_{F}\right)^{2}}} .
$$

Thus the imaginary part of the memory function is

$$
\begin{aligned}
M_{2}(\omega, T) \approx & \frac{v_{p c}}{2 \pi \hbar \varepsilon_{F} k_{F}} \int_{0}^{q_{c}} d q \frac{\left|D_{\mathbf{q}}\right|^{2} q^{2}}{\sqrt{1-\left(q / 2 k_{F}\right)^{2}}} \\
& \times\left(N\left(\omega_{\mathbf{q}}\right)+\frac{1}{2}-\frac{1}{2} \frac{\sinh \beta \hbar \omega_{\mathbf{q}}}{\cosh \beta \hbar \omega_{\mathbf{q}}+\cosh \beta \hbar \omega}\right),
\end{aligned}
$$

where the sum of Bose-Einstein and Fermi-Dirac distributions has been explicitly written with $\beta=1 / k_{B} T$. For deriving the prefactor in Eq. (4.11), the connection between the effective and total electron concentration-as outlined in Sec. III Bhas been used, together with the definition of the Fermi wave vector $k_{F}^{2}=2 \pi n$. Additionally, it is useful to write the total concentration of electrons as $n=N_{e} / v_{p c}$, where $N_{e}$ is the number of conducting electrons in the primitive cell. Upper bond $q_{c}$ can be chosen between the Debye $q_{D}$ and BlochGrüneisen $q_{B G}$ wave vector as the minimum of the two, $q_{c}=$ $\min \left\{q_{D}, q_{B G}\right\}$. For the 2D system as described in Sec. II, $q_{c}=$ $2 k_{F}$ and $q_{D}^{2}=4 \pi / v_{p c}$ and $q_{B G}<q_{D}$ if $N_{e}<1 / 2$. In further analysis we take $q_{c}=q_{D}$, since $N_{e}=1$ as stated in Sec. II. An additional simplification can be made. The square root term can be omitted in Eq. (4.11) since it has a minor influence on the integral, because $\left(q_{D} / 2 k_{F}\right)^{2}=1 / 2$ for a half-filled band. Next, Eq. (4.11) is evaluated for acoustic and optical phonons.

\section{B. Acoustic phonon memory function}

The electron-phonon coupling element Eq. (2.5) is inserted into Eq. (4.11), along with the substitution $\Omega=\hbar \omega_{\mathbf{q}}^{A}=\hbar c q$, where $c$ is the sound speed, giving

$$
\begin{aligned}
M_{2}(\omega, T) \approx & \frac{2}{\hbar\left(\hbar \omega_{D}\right)^{3}} \sqrt{\frac{2}{N_{e}}} \frac{D_{A}}{\varepsilon_{F}} \int_{0}^{\hbar \omega_{D}} \Omega^{3} d \Omega \\
& \times\left(N(\Omega)+\frac{1}{2}-\frac{1}{2} \frac{\sinh \beta \Omega}{\cosh \beta \Omega+\cosh \beta \hbar \omega}\right) .
\end{aligned}
$$

Figure 3(a) shows the imaginary part of the acoustic phonon memory function Eq. (4.12) at various temperatures. Only in certain limiting cases can Eq. (4.12) be simplified further. The temperature dependent static case is

$$
M_{2}(\omega=0, T) \approx \frac{2}{\hbar\left(\hbar \omega_{D}\right)^{3}} \sqrt{\frac{2}{N_{e}}} \frac{D_{A}}{\varepsilon_{F}} \int_{0}^{\hbar \omega_{D}} \frac{\Omega^{3}}{\sinh \beta \Omega} d \Omega,
$$

which in the $T \rightarrow 0$ limit reduces to

$$
\begin{aligned}
M_{2}(\omega=0, T \rightarrow 0) & \approx \frac{4}{\hbar\left(\hbar \omega_{D}\right)^{3}} \sqrt{\frac{2}{N_{e}}} \frac{D_{A}}{\varepsilon_{F}} \int_{0}^{\hbar \omega_{D}} \Omega^{3} e^{-\beta \Omega} d \Omega \\
& \approx \frac{24}{\hbar} \sqrt{\frac{2}{N_{e}}} \frac{D_{A}}{\varepsilon_{F}} \frac{\left(k_{B} T\right)^{4}}{\left(\hbar \omega_{D}\right)^{3}} .
\end{aligned}
$$


In the opposite, high temperature limit, it is

$$
\begin{aligned}
M_{2}(\omega & =0, T \rightarrow \infty) \\
& \approx \frac{2}{\hbar\left(\hbar \omega_{D}\right)^{3}} \sqrt{\frac{2}{N_{e}}} \frac{D_{A}}{\varepsilon_{F}} \frac{1}{\beta} \int_{0}^{\hbar \omega_{D}} \Omega^{2} d \Omega \\
& \approx \frac{2}{3 \hbar} \sqrt{\frac{2}{N_{e}}} \frac{D_{A}}{\varepsilon_{F}} k_{B} T .
\end{aligned}
$$

Also, a small but finite frequency $\omega$ correction can be included in the high $T$ limit

$$
\begin{aligned}
M_{2}(\omega & \left.\ll \omega_{D}, T \rightarrow \infty\right) \\
& \approx \frac{2}{3 \hbar} \sqrt{\frac{2}{N_{e}}} \frac{D_{A}}{\varepsilon_{F}} k_{B} T\left(1+\frac{3}{80} \frac{\left(\hbar \omega_{D}\right)^{2}(\hbar \omega)^{2}}{\left(k_{B} T\right)^{4}}\right) .
\end{aligned}
$$

The third ratio in the brackets of Eq. (4.12) can be neglected in the limit of high $\omega \gg \omega_{D}$ regardless of $T$, since $\cosh \beta \hbar \omega \rightarrow \infty$, giving

$$
\begin{aligned}
M_{2}(\omega & \left.\gg \omega_{D}, T\right) \\
& \approx \frac{2}{\hbar\left(\hbar \omega_{D}\right)^{3}} \sqrt{\frac{2}{N_{e}}} \frac{D_{A}}{\varepsilon_{F}} \int_{0}^{\hbar \omega_{D}} \Omega^{3} d \Omega\left(N(\Omega)+\frac{1}{2}\right),
\end{aligned}
$$

with the notable high $T$ limit

$$
M_{2}\left(\omega \gg \omega_{D}, T \rightarrow \infty\right) \approx \frac{1}{2 \hbar} \sqrt{\frac{2}{N_{e}}} \frac{D_{A}}{\varepsilon_{F}} k_{B} T .
$$

The finite value of $M_{2}\left(\omega \gg \omega_{D}, T\right)$ is a direct consequence of the term $N(\Omega)+1 / 2$ in Eq. (4.17) which is connected to the occupancy of the harmonic oscillator in a heat bath. At zero temperature, only quantum term $1 / 2$ remains, thus giving a finite result. This is the main difference between the memory function and the usual variation principle approach [28] to the intraband relaxation function, since the latter is always proportional to some power of $T$. In the case of $T=0$ and finite frequency, the part in the bracket in Eq. (4.12) simplifies. Then $N(\Omega)=0$ and the rest reduces to

$$
\frac{\sinh \beta \Omega}{\cosh \beta \Omega+\cosh \beta \hbar \omega} \Rightarrow \Theta(\hbar \omega-\Omega),
$$

where $\Theta$ is the Heaviside step function. Expression (4.19) changes the integration boundaries in Eq. (4.12) to finally give

$$
\begin{aligned}
& M_{2}\left(\omega<\omega_{D}, T=0\right)=\frac{1}{4} \sqrt{\frac{2}{N_{e}}} \frac{D_{A}}{\varepsilon_{F}} \frac{\omega^{4}}{\omega_{D}^{3}}=a \omega^{4}, \\
& M_{2}\left(\omega>\omega_{D}, T=0\right)=\frac{1}{4} \sqrt{\frac{2}{N_{e}}} \frac{D_{A}}{\varepsilon_{F}} \frac{\omega_{D}^{4}}{\omega_{D}^{3}}=a \omega_{D}^{4} .
\end{aligned}
$$

Thus the relaxation of conducting electrons on phonons persists at zero temperature and it follows an $\omega^{4}$ law in 2D conductors below $\omega_{D}$. Given the imaginary part of the memory function Eq. (4.12), the most elegant way to calculate its real part $M_{1}(\omega, T)$ is using the Kramers-Kronig relations. $M_{1}(\omega, T)$ is shown in Fig. 3(b) for various temperatures. $M_{1}(\omega, T)$ is noticeably linear for $\omega<\omega_{D}$, with the $T$-dependent slope $\lambda(T)$. A maximum located slightly below $\omega_{D}$ diminishes and shifts with increasing $T$. At $\omega=\omega_{D}$

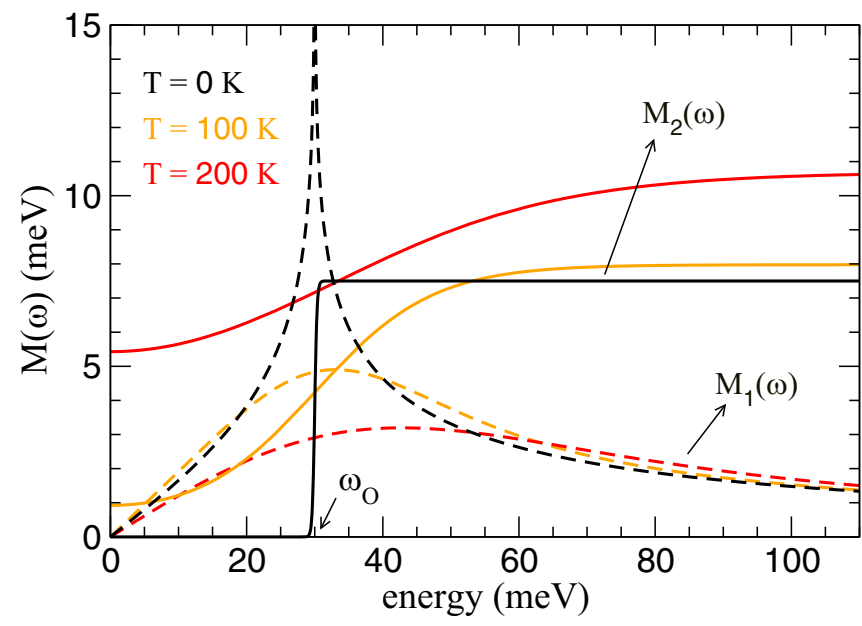

FIG. 4. Real (dashed line) and the imaginary part (solid line) of the memory function describing the scattering of an electron on an optical phonon, calculated for various temperatures. The parameters used in the calculation are $\varepsilon_{F}=0.96 \mathrm{eV}, \hbar \omega_{O}=30 \mathrm{meV}$, and $D_{O}=0.5 \mathrm{eV}$.

and $T=0$, the real part has an inflection point. For $\omega>\omega_{D}, M_{1}(\omega, T)$ is a decreasing function of $\omega$. Fortunately, $M_{1}(\omega, T=0)$ can be explicitly derived from Eq. (4.20) using the Kramers-Kronig relations

$$
\begin{aligned}
M_{1}(\omega, 0)= & \frac{2}{3} \frac{a}{\pi} \omega \omega_{D}\left(\omega_{D}^{2}+3 \omega^{2}\right) \\
& +\frac{a}{\pi}\left(\omega_{D}^{4}-\omega^{4}\right) \ln \left|\frac{\omega+\omega_{D}}{\omega-\omega_{D}}\right|,
\end{aligned}
$$

with the low and high $\omega$ expansion relative to $\omega_{D}$ :

$$
\begin{aligned}
& M_{1}\left(\omega \ll \omega_{D}, 0\right)=(8 a / 3 \pi) \omega_{D}^{3} \omega, \\
& M_{1}\left(\omega \gg \omega_{D}, 0\right)=(2 a / \pi) \omega_{D}^{5} / \omega .
\end{aligned}
$$

Expressions (4.12)-(4.22) are easily modified for the BlochGrüneisen case. It is necessary to change $\sqrt{2 / N_{e}} \rightarrow 4 N_{e}$ and $\omega_{D} \rightarrow \omega_{B G}$.

\section{Optical phonon case}

The same procedure is applied in order to determine the memory function corresponding to the optical phonon. The starting point is the equation (4.12) with the constant $\omega_{\mathbf{q}}^{O}=$ $\omega_{O}$ and a different coupling element $D_{O}$. The expression in the brackets in Eq. (4.11) is now $q$ independent

$$
M_{2}(\omega, T) \approx b\left(N\left(\omega_{O}\right)+\frac{1}{2}-\frac{1}{2} \frac{\sinh \beta \hbar \omega_{O}}{\cosh \beta \hbar \omega_{O}+\cosh \beta \hbar \omega}\right),
$$

with $b=\left(2 D_{O} \omega_{O} / 3 \varepsilon_{F}\right) \sqrt{2 / N_{e}}$. In Fig. 4, Eq. (4.24) is plotted for several temperatures.

The static case is simply

$$
M_{2}(\omega=0, T) \approx \frac{b}{\sinh \left(\beta \hbar \omega_{O}\right)}
$$

and, unlike Eq. (4.14), it has an exponential dependence $\sim \exp \left(-\omega_{O} / T\right)$ for $T \rightarrow 0$. Similar to Eq. (4.17), the $T$ - 

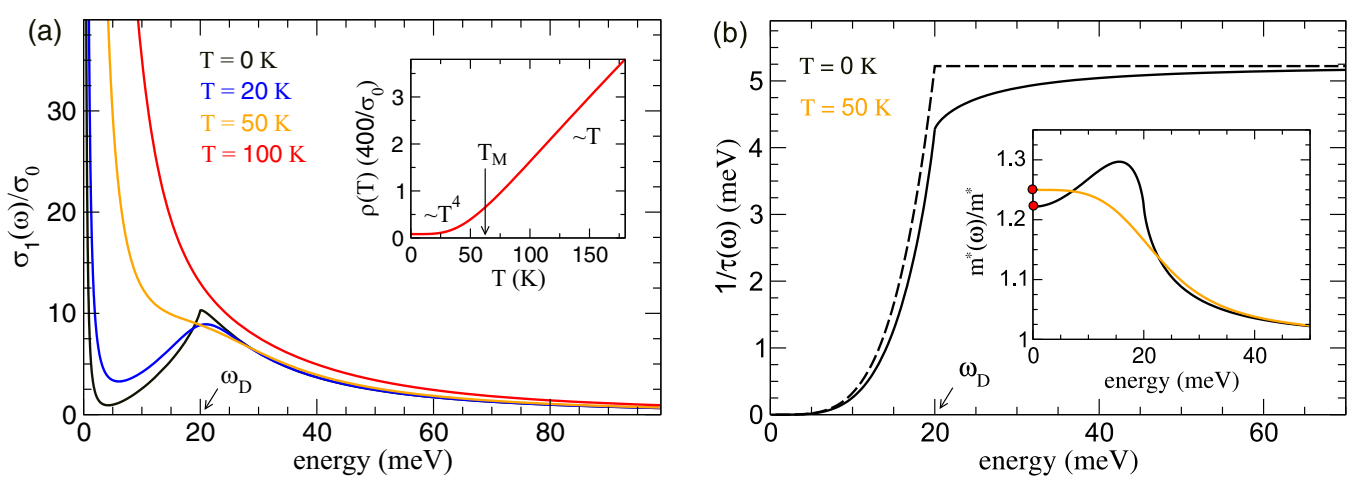

FIG. 5. (a) Real part of the dynamical conductivity Eq. (3.27) in units of $\sigma_{0}=e^{2} /(4 \hbar)$ calculated at various temperatures with the residual scattering relaxation constant $\hbar \gamma=0.1 \mathrm{meV}$. The conductivity contains the memory function for acoustic phonon Eq. (4.12) shown in Fig. 3. At $T=0$, a shark-fin shape dominates the intraband channel. The inset shows the temperature dependence of the dc resistivity. The characteristic temperature is $T_{M}=60 \mathrm{~K}$. (b) The $1 / \tau(\omega)$ at $T=0$ (solid black line) and the $M_{2}(\omega, 0)$ (dashed line) calculated from Eqs. (4.20) and (4.21). The inset shows the dynamical mass for two temperatures.

dependent high $\omega$ limit is

$$
M_{2}\left(\omega \gg \omega_{D}, T\right) \approx b\left(N\left(\omega_{O}\right)+\frac{1}{2}\right) .
$$

The $\omega$ dependence of the $T=0$ case is simple. The brackets in Eq. (4.24) reduce the step function as in Eq. (4.19)

$$
M_{2}(\omega, 0) \approx(b / 2) \Theta\left(\hbar \omega-\hbar \omega_{O}\right) .
$$

The Kramers-Kronig transformation of the above expression yields

$$
M_{1}(\omega, 0) \approx \frac{b}{2 \pi} \ln \left|\frac{\omega+\omega_{O}}{\omega-\omega_{O}}\right|,
$$

which has a singularity of the logarithmic type at $\omega=\omega_{O}$. As in the case of Eq. (4.21), this gives the simple limits in the high and low $\omega$ expansion:

$$
\begin{aligned}
& M_{1}\left(\omega \ll \omega_{O}, 0\right)=(b / \pi) \omega / \omega_{O}, \\
& M_{1}\left(\omega \gg \omega_{O}, 0\right)=(b / \pi) \omega_{O} / \omega .
\end{aligned}
$$

\section{DYNAMICAL CONDUCTIVITY OF Li(BN $)_{8}$}

Here the dynamical conductivity Eq. (3.27) is evaluated specifically for $\mathrm{Li}(\mathrm{BN})_{8}$. It is assumed that the conduction electrons are scattered only on an acoustic phonon. The scattering is described by the memory function Eq. (4.12). The dynamical conductivity Eq. (3.27) is written explicitly as

$$
\sigma(\omega, T)=\frac{i e^{2}}{m^{*}} \frac{n}{\omega+M_{1}(\omega, T)+i M_{2}(\omega, T)} .
$$

In the above expression, the connection between $n_{\alpha}$ and $n$ for the isotropic parabolic electron band is used, as described in Sec. III B. All relevant $(\omega, T)$ features of the $\sigma(\omega)$, like the height and the width of the Drude conductivity, can be traced to $M_{1}(\omega, T)$ and $M_{2}(\omega, T)$.

The real part of the dynamical conductivity is shown in Fig. 5(a) at several temperatures. A noticeable feature of the dynamical conductivity is a strong Drude peak at $\omega \approx 0$ at $T=0$. To ensure a finite width and height of the Drude peak in this limit, a constant residual scattering $\gamma$ has been introduced. This residual scattering can be justified by the presence of static disorder in the system.

The main feature in the real part of the dynamical conductivity, originating from the imaginary part of the memory function, is the shark-fin shape of the conductivity near the Debye energy $\hbar \omega_{D}$. This feature can be traced to Eq. (5.1) which, in the first approximation, gives

$$
\sigma_{1}(\omega, 0) \propto \frac{M_{2}(\omega, 0)}{\omega^{2}} .
$$

The properties of $M_{2}(\omega, 0)$, Eq. (4.20), once inserted in the above expression, determine the shark-fin shape of $\sigma_{1}(\omega)$ at $T=0$, yielding $\sigma_{1}\left(\omega<\omega_{D}\right) \propto \omega^{2}$ below and $\sigma_{1}\left(\omega>\omega_{D}\right) \propto$ $\omega^{-2}$ above the Debye frequency.

Naturally, this feature is only visible at temperatures significantly lower than the typical Debye temperature $T_{D}=230 \mathrm{~K}$ where the approximation Eq. (5.2) is valid. As seen from Fig. 5(a), already at $T \approx 0.4 T_{D}$ (red line) there are no visible features in the conductivity that could be connected to the shape of the memory function. Thus the temperature scale below which we expect to see the memory function influence on the conductivity is determined by the memory function itself. This temperature can be defined as $k_{B} T_{M}=\hbar M_{2}\left(\omega_{D}, 0\right)$, where Eq. (4.20) was used

$$
k_{B} T_{M}=\frac{D_{A}}{\sqrt{8 N_{e}}} \frac{\hbar \omega_{D}}{\varepsilon_{F}} .
$$

For parameters listed in the caption of Fig. 3, we obtain $T_{M} \approx 60 \mathrm{~K}$. This is in accordance with the results shown in Fig. 5(a), where the orange line $(T=50 \mathrm{~K})$ still has visible memory function features around $\omega_{D}$ but the red line $(T=$ $100 \mathrm{~K})$ does not.

The temperature dependent resistivity

$$
\rho(T)=M_{2}(0, T) \frac{m^{*}}{n e^{2}},
$$

as obtained from Eq. (5.1), depends entirely on $M_{2}(0, T)$. By applying Eqs. (4.13)-(4.15), a temperature dependence of resistivity $\rho(T) \propto T^{4}$ is found at low temperatures and $\rho(T) \propto T$ at high temperatures. This is in accordance with the two-dimensional Bloch law [6,49]. The resistivity Eq. (5.3) is 
shown in the inset in Fig. 5(a), where the crossing between the low and high temperature regime is roughly at $T_{M}$. Above the temperature $T_{M}$, a simple Drude model Eq. (3.8) can be used, in which the relaxation constant $\gamma(T)$ is given by Eq. (4.18). Another candidate for $\gamma$, Eq. (4.15), differs from Eq. (4.18) only by a small numerical factor. In the high $T$ limit, as seen from Fig. 3(b), the amplitude of the real part of the memory function decreases. In the first approximation, it can be discarded from the analysis of dynamical conductivity.

Very often, an extended Drude model $[23,52,53]$ is used in the analysis of the far-infrared conductivity

$$
\sigma(\omega, T)=\frac{i e^{2}}{m^{*}(\omega, T)} \frac{n}{\omega+i / \tau(\omega, T)} .
$$

Equation (5.4) is equivalent to Eq. (5.1). The newly introduced dynamic effective electron mass $m^{*}(\omega, T)$ and the dynamic relaxation time $\tau(\omega, T)$ are defined with respect to the real and imaginary part of the memory function

$$
\frac{m^{*}(\omega, T)}{m^{*}}=1+\frac{M_{1}(\omega, T)}{\omega}, \quad \frac{1}{\tau(\omega, T)}=\frac{M_{2}(\omega, T)}{1+\frac{M_{1}(\omega, T)}{\omega}} .
$$

The two quantities in (5.5) are shown in Fig. 5(b) in the acoustic phonon case. $1 / \tau(\omega)$ behaves more smoothly (black solid line) than $M_{2}(\omega)$ (black dashed line) around $\omega_{D}$, while at the low and high $\omega$ it has the same limits as $M_{2}(\omega)$. In Sec. IV B, it has been shown that the real part of the memory function is linear for small frequencies, $M_{1}(\omega \approx 0, T)=\lambda(T) \omega$. This leads in Eq. (5.5) to the increment of the bare effective mass $m^{*}(\omega=0, T) / m^{*}=1+\lambda(T)$. This feature is seen in the inset of Fig. 5(b), where the static values of the renormalized effective mass are marked with red circles.

The intraband spectral weight is conserved as it can be checked numerically

$$
\int_{0}^{\infty} \sigma_{1}(\omega, T) d \omega=\pi e^{2} n / 2 m^{*} .
$$

This is a natural consequence of the shape of the memory function conductivity Eq. (5.1) and the Kramers-Kronig relations connecting the real and the imaginary part of the memory function.

\section{CONCLUSIONS}

An equation of motion method for the electron-hole operator presented here has been successfully applied to calculate the dynamical scattering of conducting electrons on phonons in a two-dimensional free-electron-like system. The electronhole operator is written as a sum of two terms, the first of which is of the zeroth order and the second of the second order in the electron-phonon interaction. Both contributions to the electron-hole operator are evaluated using the Heisenberg equation. It is shown that the momentum and frequency dependent memory function is connected to the second term in the electron-hole propagator. The generalized Drude formula is derived with the frequency dependent memory function, which has been averaged over the Fermi surface. The imaginary part of the memory function is explicitly derived for the cases of electron scattering on an acoustic and optical phonon.
Most notable properties of the here derived memory function are a $\omega^{4}$ dependence on $T=0$ below the Debye frequency and a constant value above the Debye frequency in the case of acoustic phonon scattering. The optical phonon case is much simpler-it is a step function with the zero value below and finite above the optical phonon frequency. The real part of the memory function is obtained using the Kramers-Kronig relation to the imaginary part. It has a linear $\omega$ dependence below the Debye or optical phonon frequency, and a specific logarithmic feature around the Debye or optical frequencies. The dynamical conductivity of the lithium intercalated boron nitride shows a shark-fin shape in the vicinity of the Debye frequency for temperatures much smaller than the specific temperature $T_{M}$, defined by the memory function energy scale. Above this temperature, a simple Drude formula with a constant, temperature dependent relaxation applies. The influence of the real part of the memory function has been demonstrated in the definition of the dynamical mass in the extended Drude model where it leads to the increment of the effective mass.

\section{ACKNOWLEDGMENTS}

The author acknowledges many stimulating discussions with K. Cindrić and A. Akrap. This work was supported by the Postdoctoral Scholarship of the Swiss Confederation and in part by Swiss National Science Foundation through Project No. PP00P2_170544. Also, the hospitality of the University of Fribourg is greatly appreciated.

\section{APPENDIX A: COMMUTATORS AND ANTICOMMUTATORS}

Apart from the basic fermion and boson anticommutator and commutator

$$
\left\{c_{\mathbf{k}}, c_{\mathbf{k}^{\prime}}^{\dagger}\right\}=\delta_{\mathbf{k}, \mathbf{k}^{\prime}}, \quad\left[b_{\mathbf{k}}, b_{\mathbf{k}^{\prime}}^{\dagger}\right]=\delta_{\mathbf{k}, \mathbf{k}^{\prime}},
$$

in the Heisenberg equation, the following form is usually encountered when evaluating the bare electron and phonon energies:

$$
\begin{aligned}
{[A B, C] } & =A[B, C]+[A, C] B \\
= & A\{B, C\}-\{A, C\} B .
\end{aligned}
$$

The electron-phonon interaction term produces four operator commutators which are again decomposed to basic ones (A1):

$$
\begin{aligned}
& {[A B, C D]} \\
& \quad=A C[B, D]+A[B, C] D+C[A, D] B+[A, C] D B \\
& \quad=A\{B, C\} D-A C\{B, D\}+\{A, C\} D B-C\{A, D\} B .
\end{aligned}
$$

\section{APPENDIX B: ELECTRON SELF-ENERGY}

Here we derive the electron self-energy. To do so we search the solution of the Heisenberg equation for the fermion operator $c_{\mathbf{k}}$ :

$$
\begin{aligned}
i \hbar \frac{\partial}{\partial t} c_{\mathbf{k}} & =\hbar \omega c_{\mathbf{k}}=\left[c_{\mathbf{k}}, \hat{H}\right] \\
& =\varepsilon_{\mathbf{k}}+\frac{1}{\sqrt{N}} \sum_{\lambda} \sum_{\mathbf{q}} D_{\mathbf{q}}^{\lambda}\left(c_{\mathbf{k}-\mathbf{q}} b_{\lambda \mathbf{q}}+c_{\mathbf{k}-\mathbf{q}} b_{-\lambda \mathbf{q}}^{\dagger}\right) .
\end{aligned}
$$


The Hamiltonian in the above expression contains $H=H_{e l}+$ $H_{\mathrm{ph}}+H_{\mathrm{el}-\mathrm{ph}}$. Next, the operators on the right hand side of Eq. (B1) are evaluated. They are again inserted in the Heisenberg equation with the total Hamiltonian as in Eq. (B1). Finally, the mean field theory is used to average the boson and fermion operators. We get

$$
c_{\mathbf{k}-\mathbf{q}} b_{\lambda \mathbf{q}} \approx \frac{1}{\sqrt{N}} D_{-\mathbf{q}}^{\lambda} \frac{N\left(\omega_{\mathbf{q}}^{\lambda}\right)+1-f_{\mathbf{k}-\mathbf{q}}}{\hbar \omega-\hbar \omega_{\mathbf{q}}^{\lambda}-\varepsilon_{\mathbf{k}-\mathbf{q}}+i \eta} c_{\mathbf{k}}
$$

and similarly

$$
c_{\mathbf{k}-\mathbf{q}} b_{-\lambda \mathbf{q}}^{\dagger} \approx \frac{1}{\sqrt{N}} D_{-\mathbf{q}}^{\lambda} \frac{N\left(\omega_{\mathbf{q}}^{\lambda}\right)+f_{\mathbf{k}-\mathbf{q}}}{\hbar \omega+\hbar \omega_{\mathbf{q}}^{\lambda}-\varepsilon_{\mathbf{k}-\mathbf{q}}+i \eta} c_{\mathbf{k}} .
$$

Inserting Eqs. (B2) and (B3) back into Eq. (B1) and changing $-\mathbf{q} \rightarrow \mathbf{q}$ in the sum over $\mathbf{q}$, we get the energy equation of the fermion operator $c_{\mathbf{k}}$ :

$$
\hbar \omega c_{\mathbf{k}}=\left[\varepsilon_{\mathbf{k}}+\Sigma(\mathbf{k}, \omega)\right] c_{\mathbf{k}}
$$

In the above expression we define the electron self-energy $\Sigma(\mathbf{k}, \omega)$, which is defined as

$$
\begin{aligned}
& \Sigma(\mathbf{k}, \omega)=\sum_{\mathbf{q}} \Sigma(\mathbf{q}, \mathbf{k}, \omega) \\
& =\sum_{\mathbf{q}} \frac{1}{N} \sum_{\lambda} \sum_{s= \pm 1}\left|D_{\mathbf{q}}^{\lambda}\right|^{2} \frac{s N\left(s \omega_{\mathbf{q}}^{\lambda}\right)+s f_{\mathbf{k}+\mathbf{q}}}{\hbar \omega+s \hbar \omega_{\mathbf{q}}^{\lambda}-\varepsilon_{\mathbf{k}+\mathbf{q}}+i \eta} .
\end{aligned}
$$

[1] J. Kim, E. Yamasue, H. Okumura, and K. N. Ishihara, J. Alloys Compd. 751, 324 (2018).

[2] A. Sumiyoshi, H. Hyodo, and K. Kimura, J. Solid State Chem. 187, 208 (2012).

[3] N. H. Shimada, E. Minamitani, and S. Watanabe, Appl. Phys. Express 10, 93101 (2017).

[4] I. Lončarić, Z. Rukelj, V. M. Silkin, and V. Despoja, npj 2D Mater. Appl. 2, 33 (2018).

[5] Z. Rukelj and V. Despoja, New J. Phys. 22, 063052 (2020).

[6] N. Singh, Electronic Transport Theories, 1st ed. (CRC Press, Boca Raton, FL, 2016).

[7] R. Zwanzig, Phys. Rev. 124, 983 (1961).

[8] H. Mori, Prog. Theor. Phys. 33, 423 (1965).

[9] D. Forster, Hydrodynamic Fluctuations, Broken Symmetry, and Correlation Functions, Advanced Book Program (W. A. Benjamin, New York, 1975).

[10] W. Götze and P. Wölfle, Phys. Rev. B 6, 1226 (1972).

[11] A. Gold and W. Götze, Phys. Rev. B 33, 2495 (1986).

[12] R. Kubo, J. Phys. Soc. Jpn. 12, 570 (1957).

[13] A. Ron, Nuovo Cimento (1955-1965) 34, 1494 (1964).

[14] A. Ron, Nuovo Cimento (1955-1965) 34, 1511 (1964).

[15] G. Y. Hu and R. F. O'Connell, Phys. Rev. B 36, 5798 (1987).

[16] J. M. Luttinger and W. Kohn, Phys. Rev. 109, 1892 (1958).

[17] A. Kopeliovich, JETP 31, 323 (1970).

[18] P. M. Platzman and P. A. Wolff, Waves and Interactions in Solid State Plasmas (Academic Press, New York, 1973).

[19] K. Kechedzhi and S. Das Sarma, Phys. Rev. B 88, 085403 (2013).

[20] T. Holstein, Ann. Phys. (NY) 29, 410 (1964).

[21] P. B. Allen, Phys. Rev. B 3, 305 (1971).

[22] B. Mitrović and M. A. Fiorucci, Phys. Rev. B 31, 2694 (1985).

[23] S. V. Shulga, O. V. Dolgov, and E. G. Maksimov, Physica C 178, 266 (1991).

[24] S. G. Sharapov and J. P. Carbotte, Phys. Rev. B 72, 134506 (2005).

[25] P. B. Allen, Phys. Rev. B 92, 054305 (2015).

[26] P. Bhalla and N. Singh, Int. J. Mod. Phys. B 33, 1 (2019).

[27] G. D. Mahan, Many-Particle Physics (Springer, New York, 2000).
[28] J. M. Ziman, Electrons and Phonons: The Theory of Transport Phenomena in Solids (Oxford University Press, Oxford, 2001).

[29] E. Mariani and F. von Oppen, Phys. Rev. B 82, 195403 (2010).

[30] W. Kohn and J. M. Luttinger, Phys. Rev. 108, 590 (1957).

[31] M. Y. Reizer and A. V. Sergeev, JETP 65, 1291 (1987).

[32] A. Cano, Phys. Rev. B 79, 153410 (2009).

[33] R. B. Woolsey and R. M. White, Phys. Rev. B 1, 4474 (1970).

[34] V. S. Lutovinov and M. Y. Reizer, JETP 50, 355 (1979).

[35] W. G. Baber, Proc. R. Soc. London A 158, 383 (1937).

[36] J. Appel, Phys. Rev. 122, 1760 (1961).

[37] P. F. Maldague and C. A. Kukkonen, Phys. Rev. B 19, 6172 (1979).

[38] T. Sohier, M. Calandra, C.-H. Park, N. Bonini, N. Marzari, and F. Mauri, Phys. Rev. B 90, 125414 (2014).

[39] D. K. Efetov and P. Kim, Phys. Rev. Lett. 105, 256805 (2010).

[40] E. H. Hwang and S. Das Sarma, Phys. Rev. B 77, 115449 (2008).

[41] K. Kaasbjerg, K. S. Thygesen, and K. W. Jacobsen, Phys. Rev. B 85, 115317 (2012).

[42] T. Gunst, T. Markussen, K. Stokbro, and M. Brandbyge, Phys. Rev. B 93, 035414 (2016).

[43] D. Pines and P. Nozières, The Theory of Quantum Liquids: Normal Fermi Liquids (CRC Press, Boca Raton, 1989).

[44] D. N. Zubarev, Sov. Phys. Usp. 3, 320 (1960).

[45] G. Giuliani and G. Vignale, Quantum Theory of the Electron Liquid (Cambridge University Press, Cambridge, UK, 2005).

[46] I. Kupčić, Phys. Rev. B 91, 205428 (2015).

[47] I. Kupčić, Phys. Rev. B 95, 035403 (2017).

[48] P. B. Allen, Phys. Rev. B 17, 3725 (1978).

[49] G. Grimvall, The Electron-Phonon Interaction in Metals (North-Holland, Amsterdam, 1981).

[50] P. B. Allen and R. Silberglitt, Phys. Rev. B 9, 4733 (1974).

[51] Z. Rukelj, C. C. Homes, M. Orlita, and A. Akrap, Phys. Rev. B 102, 125201 (2020).

[52] T. Timusk, Solid State Commun. 127, 337 (2003).

[53] F. Marsiglio, T. Startseva, and J. P. Carbotte, Phys. Lett. A 245, 172 (1998). 